\title{
Multifilter photometry of Solar System objects from the SkyMapper Southern Survey ${ }^{\star}$
}

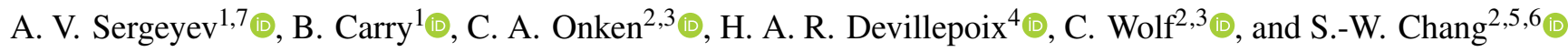 \\ ${ }^{1}$ Université Côte d'Azur, Observatoire de la Côte d'Azur, CNRS, Laboratoire Lagrange, France \\ e-mail: alexey.sergeyev@oca.eu \\ 2 Research School of Astronomy and Astrophysics, Australian National University, Canberra 2611, Australia \\ 3 Centre for Gravitational Astrophysics, College of Science, The Australian National University, ACT 2601, Australia \\ ${ }^{4}$ School of Earth and Planetary Sciences, Curtin University, Perth WA 6845, Australia \\ 5 SNU Astronomy Research Center, Seoul National University, 1 Gwanak-rho, Gwanak-gu, Seoul 08826, Korea \\ 6 Astronomy program, Dept. of Physics \& Astronomy, SNU, 1 Gwanak-rho, Gwanak-gu, Seoul 08826, Korea \\ 7 V. N. Karazin Kharkiv National University, 4 Svobody Sq., Kharkiv 61022, Ukraine
}

Received 23 August 2021 / Accepted 20 October 2021

\begin{abstract}
Context. The populations of small bodies of the Solar System (asteroids, comets, and Kuiper Belt objects) are used to constrain the origin and evolution of the Solar System. Their orbital distribution and composition distribution are both required to track the dynamical pathway from their formation regions to their current locations.

Aims. We aim to increase the sample of Solar System objects (SSOs) that have multifilter photometry and compositional taxonomy. Methods. We searched for moving objects in the SkyMapper Southern Survey. We used the predicted SSO positions to extract photometry and astrometry from the SkyMapper frames. We then applied a suite of filters to clean the catalog from false-positive detections. We finally used the near-simultaneous photometry to assign a taxonomic class to objects.

Results. We release a catalog of 880528 individual observations, consisting of 205515 known and unique SSOs. The catalog completeness is estimated to be about $97 \%$ down to $V=18 \mathrm{mag}$ and the purity is higher than $95 \%$ for known SSOs. The near-simultaneous photometry provides either three, two, or a single color that we use to classify 117356 SSOs with a scheme consistent with the widely used Bus-DeMeo taxonomy.

Conclusions. The present catalog contributes significantly to the sample of asteroids with known surface properties (about $40 \%$ of main-belt asteroids down to an absolute magnitude of 16). We will release more observations of SSOs with future SkyMapper data releases.
\end{abstract}

Key words. minor planets, asteroids: general - catalogs

\section{Introduction}

The small bodies of our Solar System (asteroids, comets, Kuiperbelt objects) are the remnants of the building blocks that accreted to form the planets. Their orbital and compositional distributions hold the record of the events that shaped our planetary system (Levison et al. 2009; DeMeo \& Carry 2014; Morbidelli et al. 2015; Clement et al. 2020).

While the number of known Solar System objects (hereafter SSOs) has increased to over a million, the fraction of SSOs with known composition remains limited. Spectroscopy in the visible and near-infrared has been used for decades to assert the composition, but the sample remains small (several thousands, e.g., Bus \& Binzel 2002; DeMeo et al. 2009; Fornasier et al. 2014; Devogèle et al. 2019; Binzel et al. 2019). On the other hand, multifilter photometry can be used to classify SSOs into broad compositional groups, providing fewer detail but large samples (up to several hundreds of thousands, e.g., Ivezić et al. 2001; Popescu et al. 2016; Sergeyev \& Carry 2021).

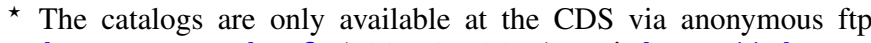
to cdsarc.u-strasbg. fr $(130.79 .128 .5)$ or via http://cdsarc. u-strasbg.fr/viz-bin/cat/J/A+A/658/A109
We analyze here the images from the SkyMapper Southern Survey (Minor Planet Centre (MPC) observatory code Q55), which uses a suite of filters that is well adapted to asteroid spectral characterization (Fig. 1), similar to those of the Sloan Digital Sky Survey (SDSS, the main source of compositional information over almost two decades; Szabó et al. 2004; Nesvorný et al. 2005; Carvano et al. 2010; DeMeo \& Carry 2013; DeMeo et al. 2014, 2019; Parker et al. 2008; Graves et al. 2018). While the SDSS completed its imaging survey in 2009, SkyMapper has started its operations in 2014 and is currently active.

The present article aims to increase the number of asteroids with multifilter photometry and taxonomy by identifying known SSOs in the SkyMapper source catalog. The article is organized as follows. In Sect. 2 we summarize the characteristics of the SkyMapper Southern Survey. In Sect. 3 we describe how we extract SSOs observations from the SkyMapper pointsource catalog, and we detail the filters we applied to the sample to reject false-positive sources in Sect. 4. The completeness and purity of the catalog is estimated in Sect. 5 . We present the catalog of SSO colors in Sect. 6 and use it to classify the SSOs consistently with the DeMeo et al. (2009) taxonomic classification in Sect. 7. We present our plan for future releases in Sect. 8. Finally, we summarize the released asteroid sample in Sect. 9. 


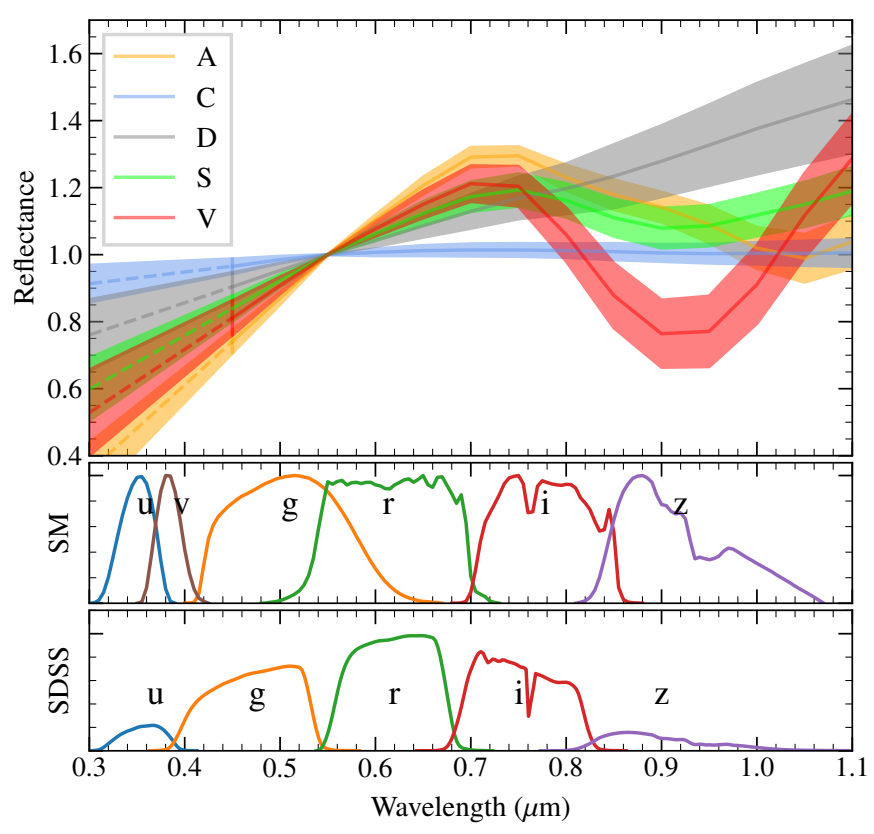

Fig. 1. Reflectance spectra of A, C, D, S, and V asteroid classes from DeMeo et al. (2009) normalized at $550 \mathrm{~nm}$. SkyMapper filters (transmission curves for a normalized quantum efficiency of the CCD are shown in SM panel) are well adapted to spectral characterization. We also report SDSS filters for comparison.

\section{SkyMapper survey}

The SkyMapper Southern Survey (SMSS) is producing a homogeneous multiband atlas of the whole southern hemisphere in $u, v, g, r, i, z$ filters (Wolf et al. 2018). Observations were carried out with a $1.35 \mathrm{~m}$ telescope located at Siding Spring Observatory (IAU code Q55). The telescope has an $\mathrm{f} / 4.8$ focal ratio and is equipped with a mosaic CCD camera with 268 million pixels. The third data release (DR3) covers an area of more than $24000 \mathrm{deg}^{2}$ and contains over 200000 images with over eight billion individual source detections (see the SkyMapper website ${ }^{1}$ and the DR2 release article for details: Onken et al. 2019). The data in DR3 were obtained between March 2014 and October 2019. Individual deep exposures can reach magnitudes of 20 in $u, v, z, 21$ in $i$, and 22 in $g, r(10 \sigma$ detections $)$ in the AB system (Oke \& Gunn 1983). The median seeing ranges from 3.3" in $u$ to $2.5^{\prime \prime}$ in $z$.

The SMSS covers each field on the sky in three primary modes: a shallow six-filter sequence with exposure times between 5 and $40 \mathrm{~s}$ that reaches depths of 18 ABmag, a deep 10image sequence of uvgruvizuv with 100-s exposures, and pairs of deep exposures in $g r$ and $i z$. This observing strategy, in conjunction with the enhanced sensitivity of $g, r$, gives rise to a predominance of $g-r$ colors in the results presented in Sect. 6, but almost always leads to the measurement of at least one photometric color obtained with $\lesssim 2 \mathrm{~min}$ between exposure midpoints in the two filters.

The work presented here adopts a single photometric measurement associated with each SkyMapper image, although for objects with significant motion during the exposure, additional information about shape and rotation parameters may be available from a more detailed analysis (e.g., the SkyMapper observations of the Earth-impacting 2018 LA, Jenniskens et al. 2021).

\footnotetext{
1 See https://skymapper.anu.edu.au
}

We present a typical suite of images illustrating the apparent motion of SSOs in SkyMapper frames (Fig. 2).

\section{Extracting candidate SSOs}

For each of the 208860 images contained in SMSS DR3, we compiled all known SSOs that might be present in the images by performing a search with SkyBoT (Berthier et al. 2006, 2016), a Virtual Observatory Web Service providing a cone-search utility for SSOs. The $2^{\circ}$ cone-search radius we used is slightly larger than the $1.7^{\circ}$ center-to-corner size of the SkyMapper camera and returned 43501635 predicted SSO locations with no initial filtering with a magnitude limit. Of these, 16915791 are predicted within the field of view (FoV). The smaller number in the number of SSO-predicted locations is explained by the search and difference in FoV area.

For each predicted SSO position, we extracted all sources listed in the SMSS DR3 photometry table ${ }^{2}$ within a radius of twice the SkyBoT-reported position uncertainty (with a floor of $\left.5^{\prime \prime}\right)$. We obtained a list of 9367950 SkyMapper sources that are associated with the 2047588 predicted SSO positions.

\section{Rejecting false-positive sources}

The fraction of interlopers among the 9367950 sources extracted above is large. First, multiple SkyMapper sources may be located by mere chance in close vicinity to predicted SSO positions. Second, many SSOs are too faint to have been imaged by SkyMapper, and the extracted sources correspond to spurious associations. We thus applied two filters to the extracted sources to reject false-positive SSOs associations.

\subsection{Comparison with Gaia}

We first identify stationary sources (stars, unresolved galaxies) by comparison with the catalog of sources with Gaia DR2 (Brown et al. 2018). We find 8203916 Gaia sources within 2" (smaller than the median SkyMapper seeing, Sect. 2) of the extracted sources. Although this certainly includes some real associations with observed SSOs, we removed them from the catalog to avoid introducing biases in the measured photometry.

\subsection{Comparison with the expected photometry}

We present in Fig. 3 a comparison of the predicted $V$ magnitudes of SSOs with SkyMapper-measured magnitudes. These magnitudes are in almost all cases the point spread function (PSF) photometry. However, owing to the apparent motion of SSOs during an exposure, the PSF photometry may underestimate the true magnitude of the observed SSO. We computed this underestimation as a function of exposure time and SSO apparent velocity. We report PSF magnitudes for most SSO observations, only reporting Petrosian magnitudes for observed SSOs with an apparent velocity of more than $50^{\prime \prime} \mathrm{h}$ and image exposure time of $100 \mathrm{~s}$. PSF photometry was preferred over Petrosian overall because its accuracy in the case of nontrailed sources is higher.

Many contaminants are still present and are easily identified by their large magnitude difference with respect to the predicted magnitudes. These contaminants correspond to SSOs that are too faint to have been detected and where erroneously associated with stationary sources.

2 The SMSS photometry table contains the per-image measurements, as opposed to the averaged quantities in the master table. 

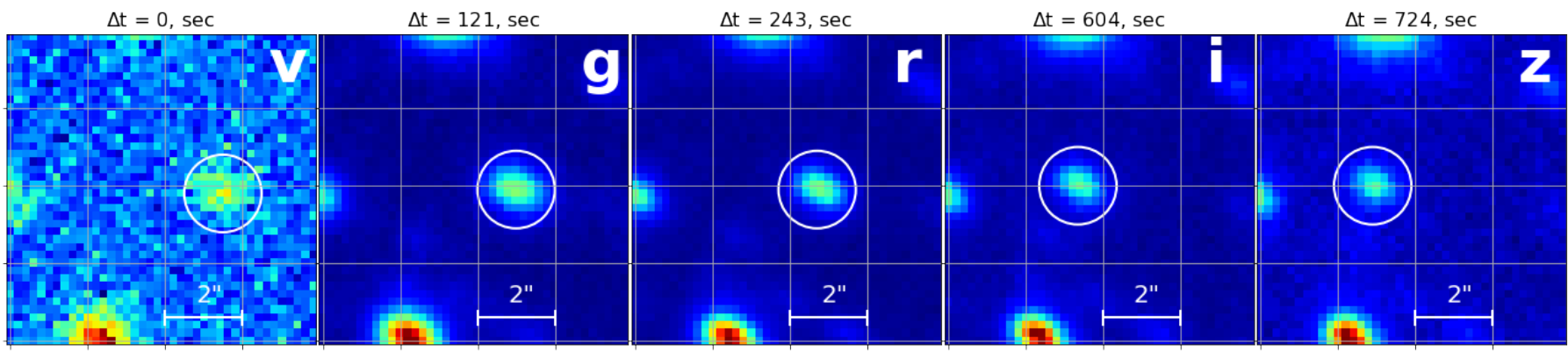

Fig. 2. SkyMapper multicolor observations of SSOs, here the asteroid (4365) Ivanova. The time interval between each frame and the first one (here $v$ ) is reported above each image. North is up and east is left.
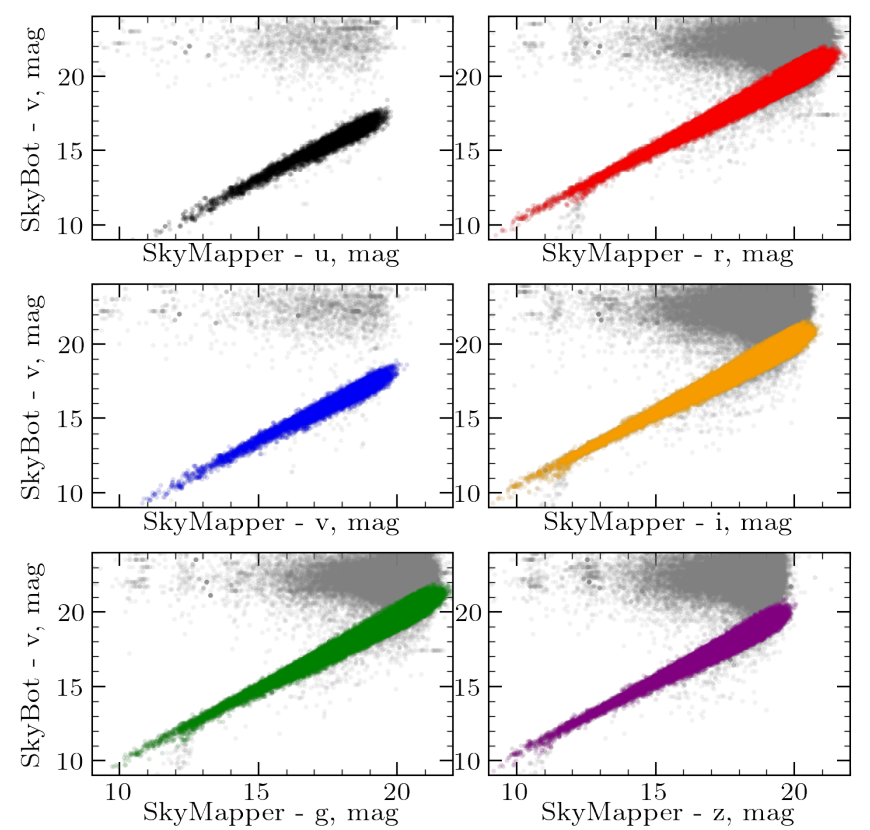

Fig. 3. Measured SkyMapper magnitude in each filter compared with the predicted $V$ magnitude of SSOs (colored dots). The light gray dots correspond to rejected sources, spuriously associated to SSOs that are too faint to have been detected (see text).

As these contaminants tend to be located at large angular separation from the predicted SSO position, we rejected all sources farther than $5^{\prime \prime}$ from SkyBoT prediction (which also implies a high SkyBoT position uncertainty). The next cleaning procedure was based on the SkyBoT and SkyMapper magnitude difference. We compared visual magnitudes as predicted by SkyBoT with the SkyMapper sample and calculated regression slopes and offsets for each filter, which are presented in Table 1. Then we removed sources showing a difference in magnitude minus the photometry magnitude error larger than $1.0 \mathrm{mag}$. A few observed SSOs appear to have saturated ( $g, r, i$ filters), with magnitudes $\lesssim 10$ in shallow images or $\lesssim 13.8,13.8$, and 13 in deeper images, and the latter condition removes them as well.

Finally, whenever several SkyMapper sources remained associated with a SSO, we selected the closest and rejected the others. After these filtering steps, we obtained a list of 880528 measurements of 205515 individual SSOs. We present their distribution on the sky in Fig. 4.

The mean difference between predicted and measured SSO positions is -0.014 and -0.075 arcsec with standard deviations of 0.15 and 0.11 arcsec for RA and Dec respectively (Fig. 5),
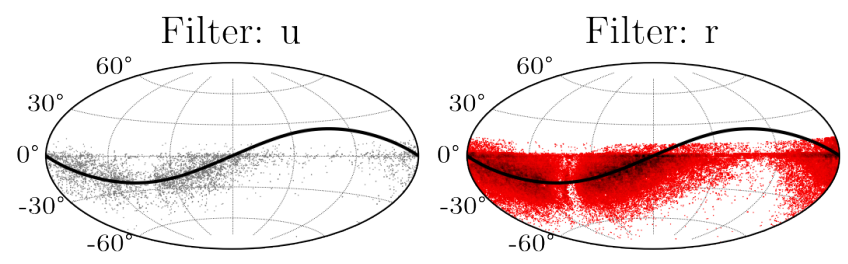

Filter: $\mathrm{v}$

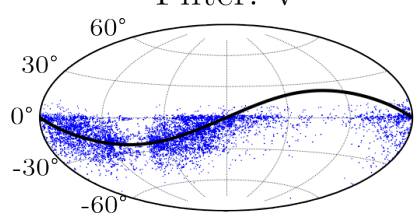

Filter: i

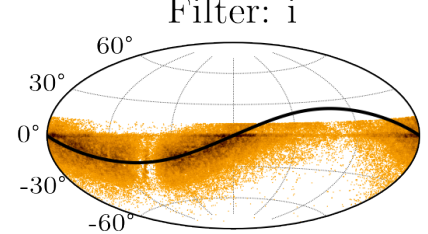

Filter: g
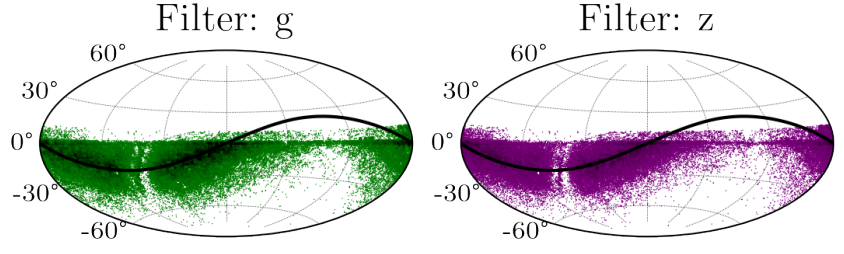

Fig. 4. Sky distribution (equatorial frame) of 880528 SkyMapper SSO observations in each filter. The black curve represents the ecliptic plane.

Table 1. Average values of the slope and magnitude offset between SkyBoT SSOs visual magnitude and each SkyMapper band magnitude.

\begin{tabular}{lrrr}
\hline \hline Filter & Slope & Offset & \# $_{\text {SSOs }}$ \\
\hline$u$ & 0.927 & -0.776 & 2850 \\
$v$ & 0.937 & -0.550 & 5302 \\
$g$ & 0.976 & 0.133 & 129789 \\
$r$ & 0.973 & 0.571 & 151809 \\
$i$ & 0.971 & 0.814 & 125395 \\
$z$ & 0.968 & 0.837 & 62774 \\
\hline
\end{tabular}

which is well below the typical seeing of SkyMapper images. Although the mean number of observations for an individual SSO is about four, hundreds of asteroids have dozens of observations, while most of them were observed only once or twice: Fig. 6. We present the distribution of measurements of the dynamical classes in Table 2 and describe the catalog of measurements in Appendix A. 

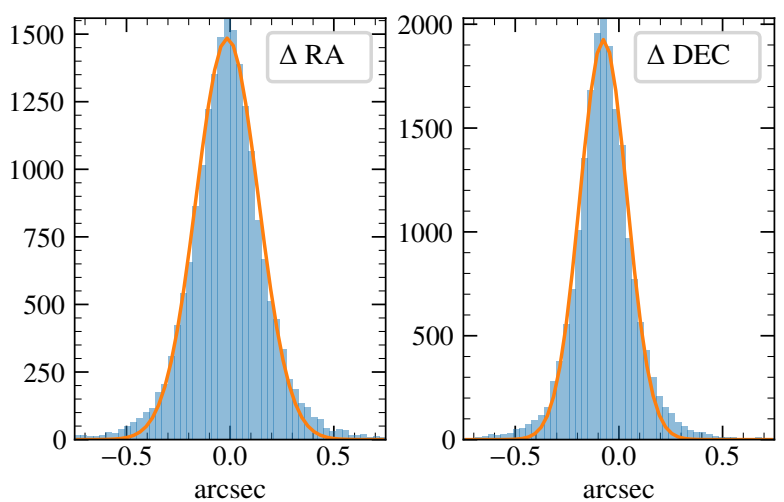

Fig. 5. Distribution of the coordinate difference between SkyMapper sources and predicted SkyBoT SSO positions.

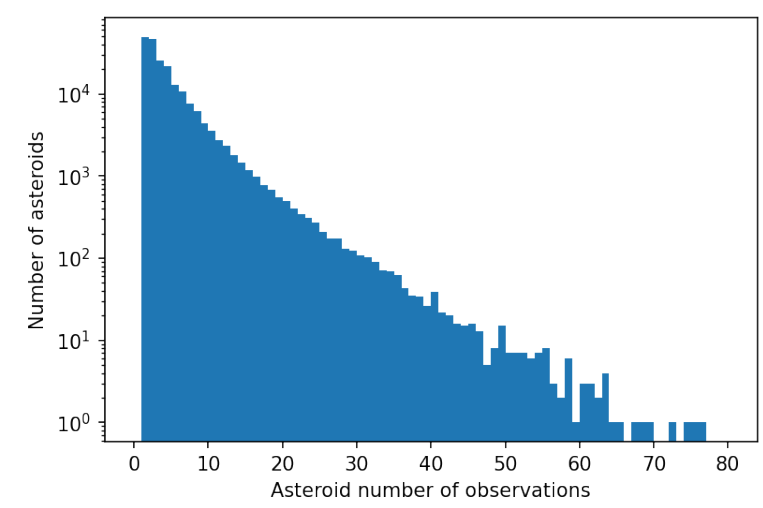

Fig. 6. SkyMapper distribution of asteroid observations.

Table 2. Number of observations $\left(N_{\mathrm{obs}}\right)$ of $N_{\mathrm{obj}}$ unique objects, sorted by dynamical classes.

\begin{tabular}{lrr}
\hline \hline Dynamical class & $N_{\text {obj }}$ & $N_{\text {obs }}$ \\
\hline NEA $>$ Aten & 39 & 103 \\
NEA $>$ Apollo & 282 & 822 \\
NEA $>$ Amor & 348 & 1289 \\
Mars-Crosser & 2487 & 9807 \\
\hline Hungaria & 4035 & 13803 \\
MBA $>$ Inner & 65231 & 287664 \\
MBA $>$ Middle & 71677 & 301935 \\
MBA $>$ Outer & 57721 & 245664 \\
MBA $>$ Cybele & 811 & 4481 \\
MBA $>$ Hilda & 829 & 4298 \\
\hline Trojan & 1928 & 10103 \\
Centaur & 24 & 127 \\
KBO & 37 & 189 \\
Comet & 65 & 226 \\
Planet & 1 & 17 \\
\hline Total & 205515 & 880528 \\
\hline
\end{tabular}

\section{Purity and completeness}

The completeness indicates the fraction of reported SSO observations with respect to how many SSOs were present in the FoV. The purity indicates the fraction of contamination among the released observations. For both estimators, the closer to unity the better. We estimated the completeness by comparing the number

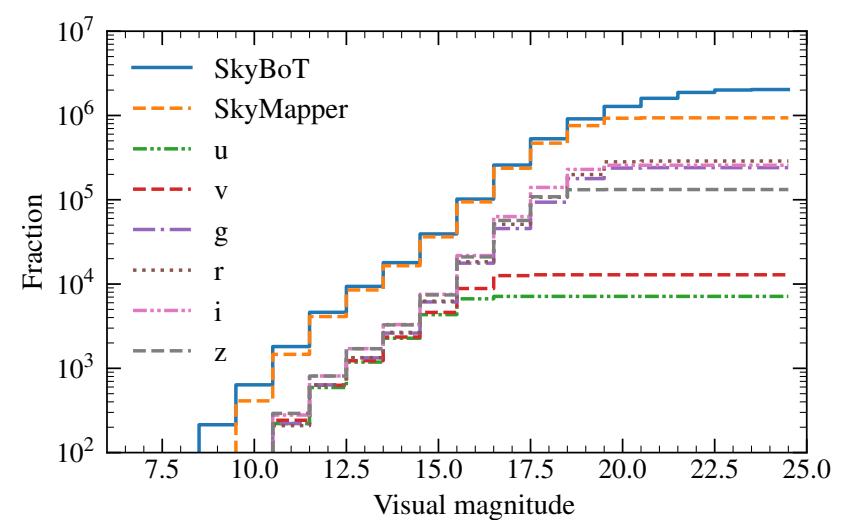

Fig. 7. Completeness as a function of predicted $V$ magnitude for the whole catalog (SkyMapper), and for each filter.

of SSO observations predicted by SkyBoT with the number of sources after filtering.

At face value (16915791 versus 880528 ), the completeness is only $5 \%$. Most nondetected SSOs, however, are simply those beyond the SkyMapper FoV or are too faint to be detected. Thus the completeness of the sources inside the CCD field of view for $V$ between 11 and 18 mag is above $97 \%$. (Fig. 7)

We estimated the purity through a visual inspection of frames centered on SSO positions in both SkyMapper and Panoramic Survey Telescope And Rapid Response System (Pan-STARRS) archives. We generated cutout frames using the SkyMapper image cutout service ${ }^{3}$, and downloaded Pan-STARRS cutout stacked images at the same coordinates (Chambers et al. 2016). The Pan-STARRS survey has a significantly deeper limiting magnitude. Thus, all stationary SkyMapper sources should be visible in Pan-STARRS images. As the Pan-STARRS survey did not image the sky below a declination pf $-30^{\circ}$, we tested the purity for sources located north of this declination only.

We inspected all available 189 Kuiper belt objects (KBO) observations, 1683 near-earth asteroids (NEA), and 1000 randomly selected main belt asteroids (MBA) observations with a declination greater than $-30 \mathrm{deg}$. The purity of the SkyMapper SSOs survey is about $100 \%$ down to magnitude $V \approx 20$, where it begins to drop (Table 3).

\section{Computation of colors}

We built the colors of the detected SSOs for compositional (taxonomic) purposes. Owing to the intrinsic photometric variability of asteroids caused by their irregular shape (Szabó et al. 2004; Popescu et al. 2016; Carry 2018), color indices have to be calculated from near-simultaneous observations. We only considered observations taken within a maximum of 20 minutes of each other. Although this threshold may appear large, it mainly affects $u$ and $v$ filter combinations. The majority (95+\%) of $g-r$ and $i-z$ colors were acquired within $2 \mathrm{~min}$, and $r-i$ colors were obtained in 6 min (Fig. 8).

Szabó et al. (2004) analyzed the color variability of asteroids from the 4th SDSS Moving Object Catalog (MOC4). With conservative assumptions, they showed that 5-min time differences have an effect on the color of less than $0.03 \mathrm{mag}$. Therefore the typical 2-min difference in our $g-r$ and $i-z$ data will not have a significant effect on the color estimation.

3 See https://skymapper.anu.edu.au/how-to-access/ 
Table 3. Purity (expressed in percent) of MBAs, NEAs, and KBOs as a function of their apparent $V$ magnitude.

\begin{tabular}{ccccccc}
\hline \hline $\begin{array}{c}V \\
(\mathrm{mag})\end{array}$ & $N_{\mathrm{MBA}}$ & $N_{\text {NEA }}$ & $N_{\text {KBO }}$ & $\begin{array}{c}\text { MBA } \\
(\%)\end{array}$ & $\begin{array}{c}\text { NEA } \\
(\%)\end{array}$ & $\begin{array}{c}\text { KBO } \\
(\%)\end{array}$ \\
\hline 10 & 0 & 0 & 0 & - & - & - \\
11 & 2 & 5 & 0 & 100 & 100 & - \\
12 & 2 & 0 & 0 & 100 & - & - \\
13 & 4 & 23 & 0 & 100 & 100 & - \\
14 & 6 & 5 & 0 & 100 & 100 & - \\
15 & 14 & 23 & 49 & 100 & 100 & 100 \\
16 & 40 & 83 & 0 & 100 & 99 & - \\
17 & 105 & 247 & 0 & 100 & 100 & - \\
18 & 174 & 321 & 14 & 100 & 100 & 100 \\
19 & 289 & 421 & 29 & 100 & 100 & 100 \\
20 & 298 & 418 & 54 & 99 & 99 & 100 \\
21 & 63 & 133 & 30 & 94 & 93 & 97 \\
22 & 2 & 4 & 4 & 50 & 100 & 75 \\
23 & 0 & 0 & 0 & - & - & - \\
\hline
\end{tabular}

Notes. We also list the number of sources $(N)$ that we visually verified in each magnitude bin.

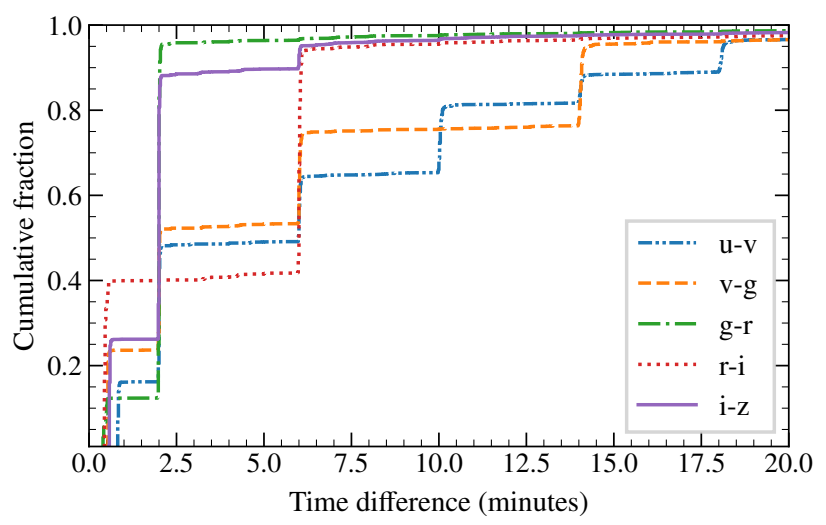

Fig. 8. Cumulative histogram of the time difference between filters.

We provide the catalog of 669545 measured colors of 139220 SSOs, specifying the time difference between each acquisition. We describe the catalog elements in Appendix A.

Some asteroid colors were measured multiple times. We therefore calculated the weighted mean color value of individual SSOs, taking the magnitude uncertainty and the time difference between observations and set weights into account as $1 / \operatorname{mag}_{\text {err }}+0.1 / \Delta d$, where $\Delta d$ is the time difference in days while $\operatorname{mag}_{\mathrm{err}}=\sqrt{\operatorname{mag} 1_{\mathrm{err}}^{2}+\operatorname{mag} 2_{\mathrm{err}}^{2}}$ is the color uncertainty of two photometry measurements mag1, mag2. If the SSO had multiple color measurements the error was computed as the weighted mean value of the color uncertainties. We also computed 13783 colors that were not directly observed, but are combinations of measured colors: for instance, we computed $r-z$ from $g-r$ and $g-z$.

As a result, we constructed a catalog of 139220 SSOs which contain at least one measured color. The total number of unique SSO colors is 388943 , both observed and derived (Table 4).

In order to determine the time-lag effect on the SkyMapper asteroids colors, we estimated the dependence of the brightness variability on the asteroid rotation period. We downloaded
Table 4. Number of measured colors $\left(N_{\text {mes }}\right)$ for each pair of filters, associated with $N_{\text {obj }}$ unique SSOs.

\begin{tabular}{lrrr}
\hline \hline Color & $N_{\text {mes }}$ & $N_{\text {obj }}$ & $N_{\text {comp }}$ \\
\hline$g-r$ & 201910 & 117356 & 205 \\
$i-z$ & 130065 & 57735 & 186 \\
$r-i$ & 86518 & 58626 & 1425 \\
$g-i$ & 74693 & 54759 & 2737 \\
$r-z$ & 55560 & 36689 & 3383 \\
$g-z$ & 48290 & 34333 & 4066 \\
$u-v$ & 12028 & 2628 & 26 \\
$v-g$ & 9963 & 4316 & 211 \\
$v-r$ & 9813 & 4317 & 245 \\
$v-z$ & 9461 & 4241 & 306 \\
$v-i$ & 9396 & 4254 & 331 \\
$u-g$ & 5620 & 2432 & 139 \\
$u-r$ & 5551 & 2434 & 148 \\
$u-z$ & 5363 & 2410 & 176 \\
$u-i$ & 5314 & 2413 & 199 \\
\hline Total & 669545 & 388943 & 13783 \\
\hline & & &
\end{tabular}

Notes. We also report the number of colors computed from linear combination of colors $\left(N_{\text {comp }}\right.$, see text).

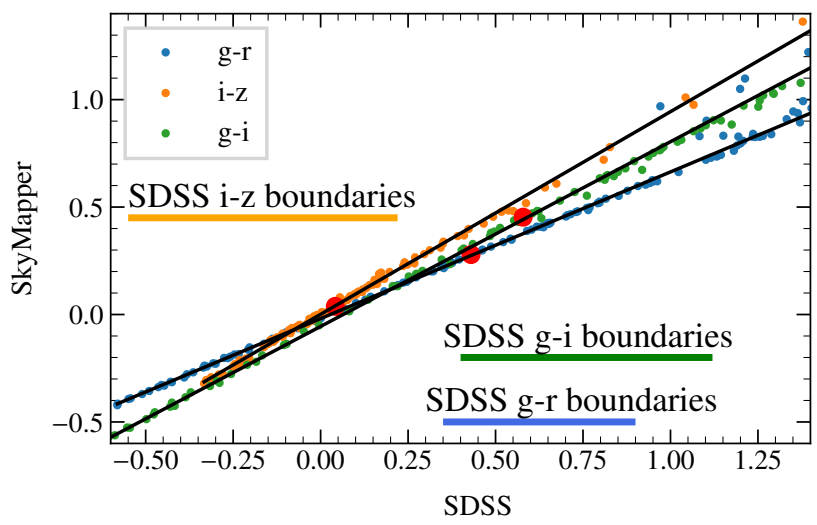

Fig. 9. $g-r$ (blue), $g-i$ (green), and $i-z$ (orange) colors from the SDSS compared with SkyMapper for a wide range of stellar classes. Red dots represent the colors of the Sun (Holmberg et al. 2006). The colored horizontal lines illustrate the range of colors for the SDSS asteroid taxonomy (Sergeyev \& Carry 2021).

the latest data release from the Asteroid Lightcurve Database ${ }^{4}$, which contains periods and amplitudes of more than 30000 known SSOs. Then we compared SkyMapper data and selected joint asteroids. We obtained samples of more than 12000 asteroids with $g-r$ and $i-z$ colors and almost 8000 asteroids with $g-i$ colors. For each asteroid in the samples, we calculated the maximum expected color change arising from asteroid rotation between the SkyMapper imaging epochs (still limited to a 20min window) as the product of the photometric amplitude and the number of half-periods represented by the SkyMapper time difference.

The magnitude difference in our samples from the rotation effect was less than the color uncertainties for 96,97 , and $87 \%$ of asteroids in $g-r, i-z$, and $g-i$ colors. We therefore consider the overall impact of rotation on the colors we report to be small.

4 See https://minplanobs.org/MPInfo/php/lcdb.php 

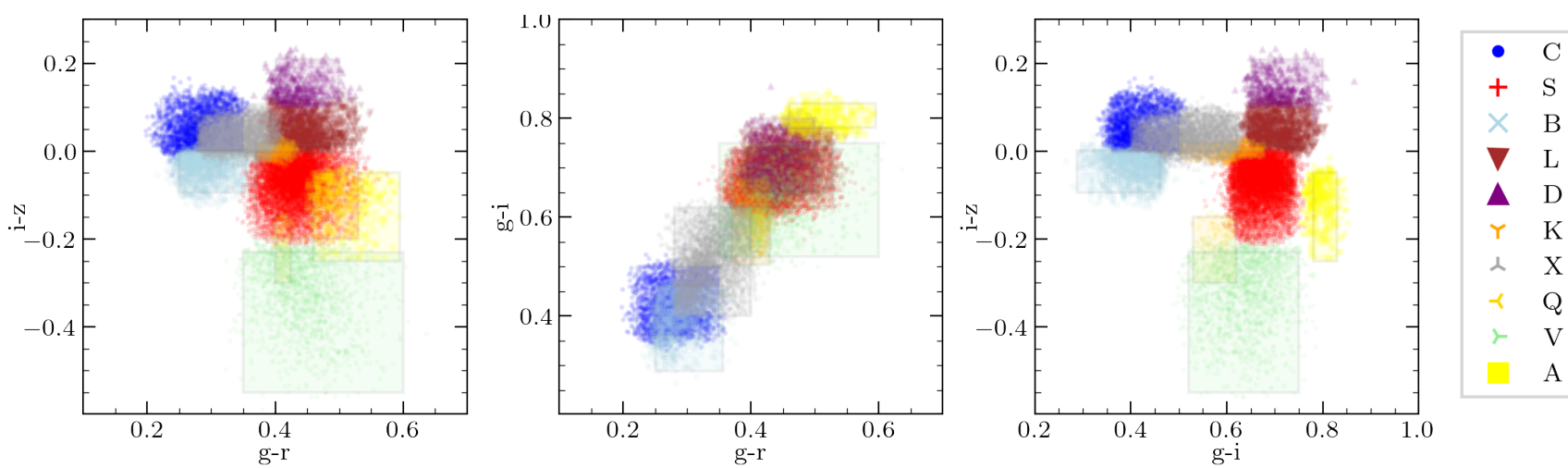

Fig. 10. Taxonomy of 29779 SkyMapper asteroids with a probability higher than 0.2 with three colors. Boxes show the boundaries of the taxonomic classes. Color points mark individual asteroids.
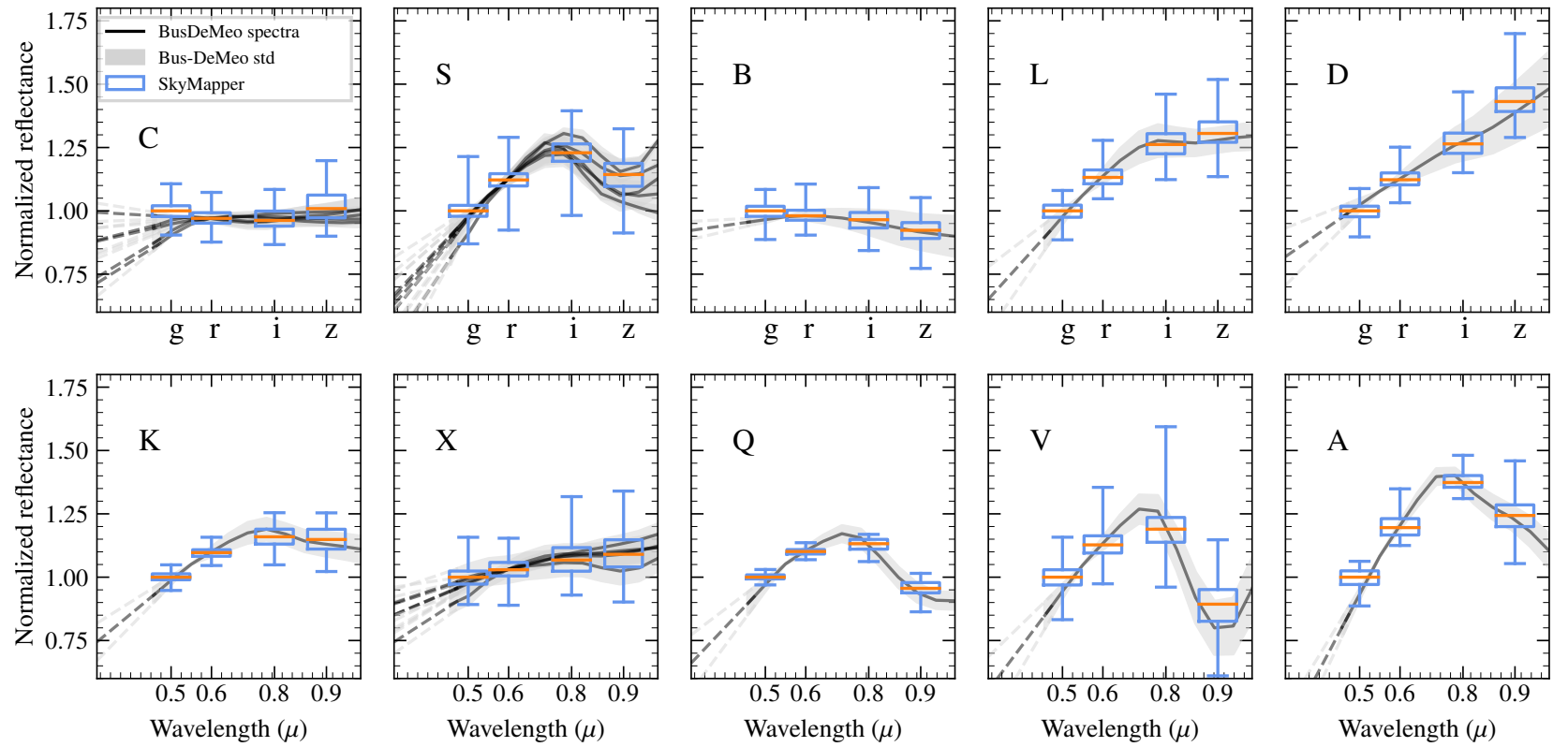

Fig. 11. Pseudo-reflectance spectra of asteroids based on their $g-r, g-i$, and $i-z$ colors. We indicate the average wavelength of each filter in the lower plots. The distribution of values for each band is represented by whiskers (95\% extrema, and the 25, 50, and 75\% quartiles). For each class, we also represent the associated template spectra of the Bus-DeMeo taxonomy (DeMeo et al. 2009).
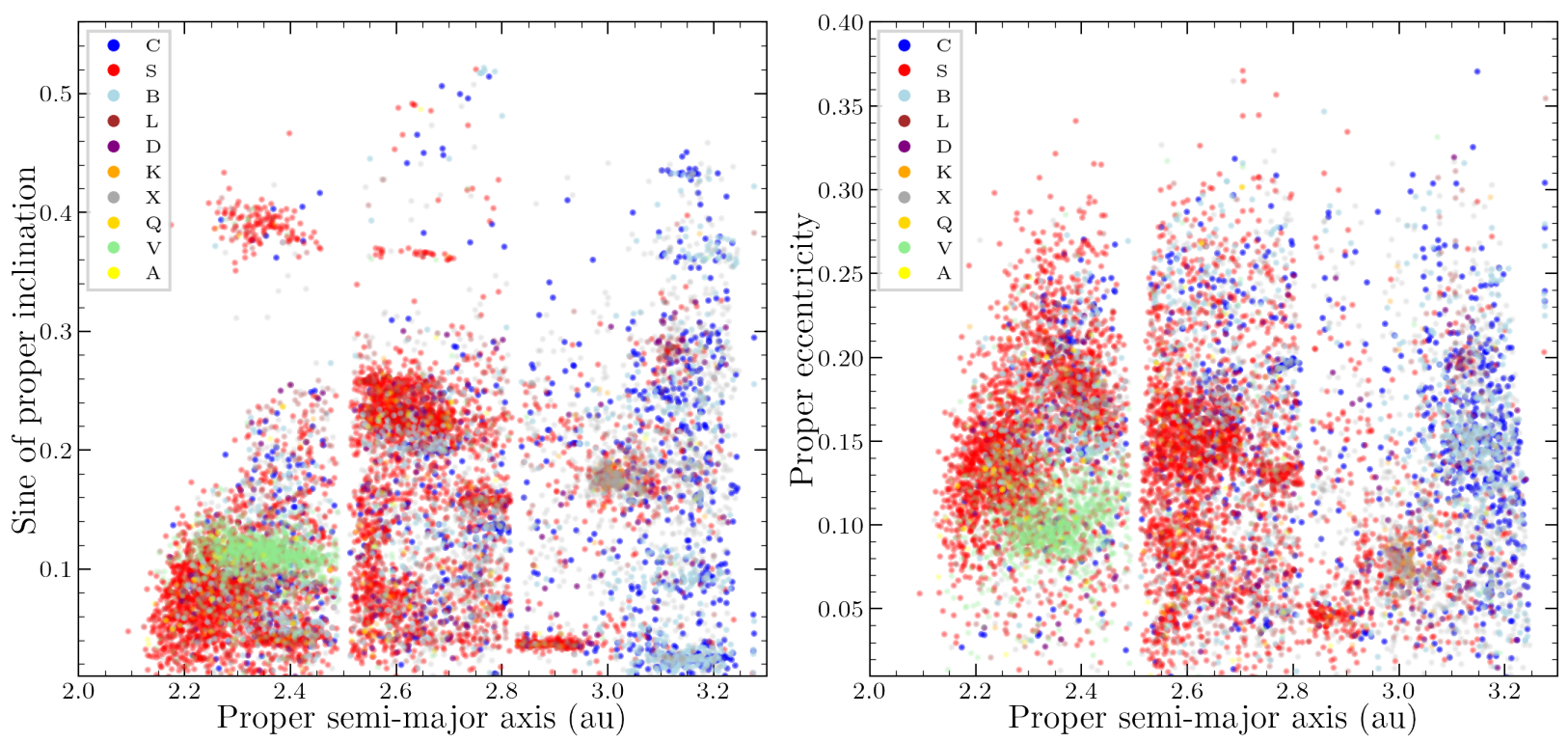

Fig. 12. Orbital distribution of the SSOs, color-coded by taxonomic class based on their $g-r, g-i$, and $i-z$ colors. 


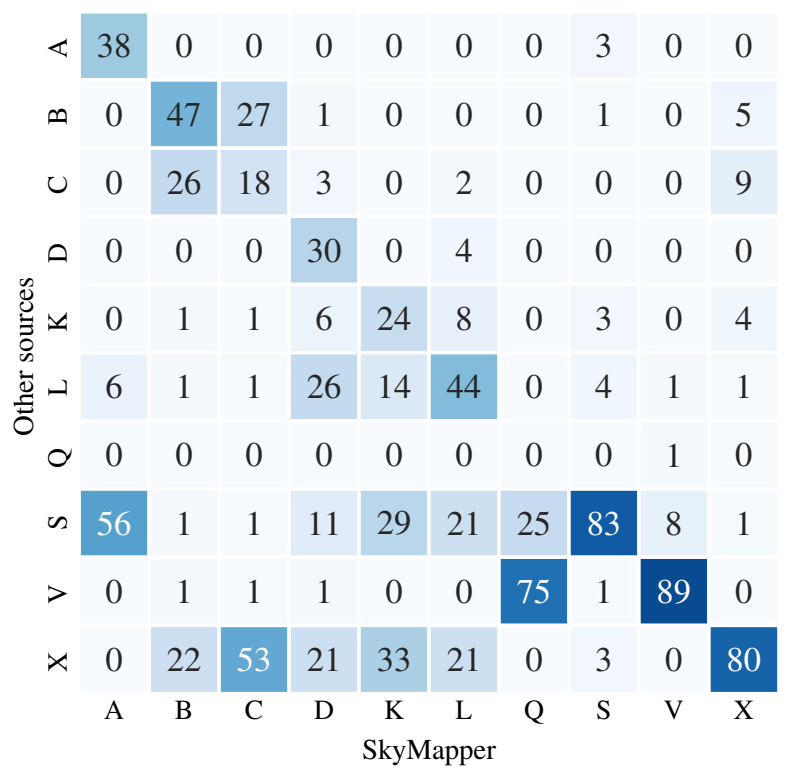

Fig. 13. Confusion matrix of the taxonomy of 1697 obtained by spectra asteroids and from Skymapper observations based on their $g-r, g-i$, and $i-z$ colors. The values are reported in percent.

\section{Taxonomy}

We used multicolor photometry to classify asteroids into a scheme consistent with the widely used Bus-DeMeo taxonomy (DeMeo et al. 2009), in which asteroid color values are used to define a certain taxonomy complex. Following our recent work with the SDSS (Sergeyev \& Carry 2021), we used a modified version of the approach of DeMeo \& Carry (2013), in which a decision tree based on colors is used to assign the taxonomic class. Where, for the each asteroid, we calculate the probability of it being associated with each taxonomic broad complex (A, B, C, D, K, L, Q, S, V, and X). We computed the intersection between the volume occupied by each taxonomy complex and the color(s) of the asteroid, represented as a $n$-dimensional Gaussian probability density function based on color values and its uncertainties. (Sergeyev \& Carry 2021).

\subsection{SkyMapper taxonomy boundaries}

We first converted the color ranges of each taxonomic class (DeMeo \& Carry 2013) from SDSS into SkyMapper filters. We used the color coefficients of Casagrande et al. (2019) and Huang et al. (2019). We compared the $g-r, g-i$, and $i-z$ values from SkyMapper with those from the SDSS for a wide range of stellar classes (Fig. 9, data from Pickles 1998). We fit the colorcolor dependence by linear regression using the Siegel (1982) approximation. These linear coefficients were used to convert the SDSS color boundaries into the SkyMapper photometric system.

\subsection{Multicolor based taxonomy}

Owing to the survey strategy, not all SSOs have the same suite of colors (Table 4). We therefore adapted our approach (Sergeyev \& Carry 2021) to handle both three-color $(g-r, g-i$, $i-z)$ and two-color $-(g-r, i-z),(g-i, i-z)$, or $(r-i, i-z)$ cases. We restricted the list of pairs of colors to those containing $i-z$ : it probes the $1 \mu \mathrm{m}$ absorption band which is one of the most characteristic spectral features in asteroid taxonomies (Chapman et al. 1975). We also shrank the boundaries to more stringent ranges for the cases with two colors only.
Table 5. Number of asteroids in each taxonomic complex for different color sets.

\begin{tabular}{crrrrr}
\hline \hline Complex & $(g r, g i, i z)$ & $(g r, i z)$ & $(g i, i z)$ & $(r i, i z)$ & $(g r)$ \\
\hline A & 537 & 309 & 947 & 4551 & \\
B & 1914 & 4587 & 2760 & 1317 & \\
C & 2180 & 6175 & 2735 & 3554 & 40573 \\
D & 466 & 901 & 1544 & 1404 & \\
K & 1005 & 1254 & 2661 & 882 & \\
L & 1709 & 3611 & 1275 & 973 & \\
Q & 164 & 633 & 845 & 532 & \\
S & 8256 & 13753 & 10577 & 10608 & 76783 \\
V & 1787 & 1125 & 843 & 1573 & \\
X & 4876 & 2398 & 4448 & 5479 & \\
U & 9882 & 4317 & 4664 & 4625 & \\
\hline Total & 32776 & 39063 & 33299 & 35498 & 117356 \\
\hline
\end{tabular}

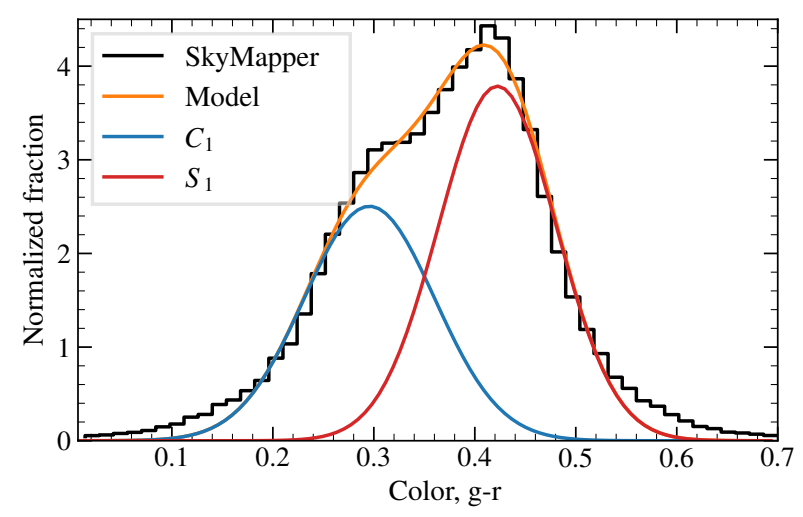

Fig. 14. $g-r$ color distribution of SkyMapper asteroids (black) were fit by the sum of two normal distributions (orange) that could be associated with $C_{1}$ (opaque-rich, blue) and $S_{1}$ (mafic-silicate rich, red) asteroid complexes.

For each observation, we computed the volume it occupies in the color space (either 2D or 3D) based on the corresponding Gaussian distribution, whose $\sigma$ were set to color uncertainties. We then computed a score for each class, $\mathcal{P}_{k}$, based on the volume of the intersection between the volume of each observation and the space occupied by each taxonomic complex (Fig. 10), normalized by the volume of the Gaussian:

$V_{\sigma}=\prod_{j=1}^{N}\left(\operatorname{erf}\left[\frac{b_{j}-\mu_{j}}{\sqrt{2} \sigma_{j}}\right]-\operatorname{erf}\left[\frac{a_{j}-\mu_{j}}{\sqrt{2} \sigma_{j}}\right]\right)$,

where $\operatorname{erf}(z)$ is the error function, $\operatorname{erf}(z)=\frac{2}{\sqrt{\pi}} \int_{0}^{z} e^{-t^{2}} \mathrm{~d} t$, the index $j$ indicates the colors (with $N \in\{2,3\}$ ), $a_{j}$ and $b_{j}$ are the color boundaries of the complexes, and $\mu_{j}$ and $\sigma_{j}$ are the color and uncertainty of the SSO. Hence, for a given observation, the volumes of all intersections sum to one. These normalized volumes correspond to the probabilities $\mathcal{P}_{k}$ of pertaining to each taxonomic class.

We then assigned to each object its most probable class. The only exception to this rule is the unknown class (labeled U), which was assigned only if its probability was strictly equal to 1. Otherwise, whenever $U$ is the most probable but not equal to unity, we assigned the second most-probable class. As an example, we present in Fig. 10 the distribution in the threecolor space of 29779 asteroids for which the probability is above 

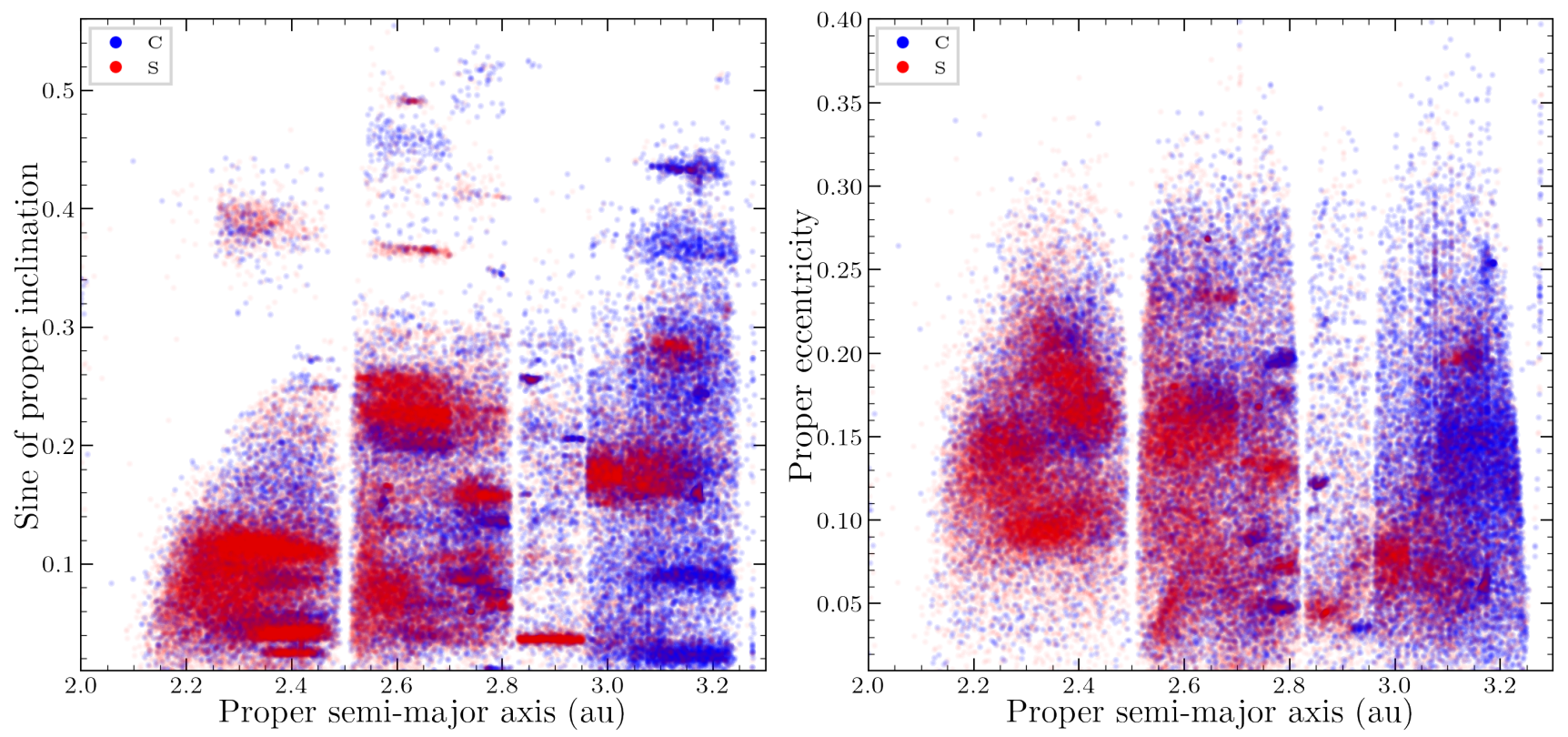

Fig. 15. Orbital distribution of the SkyMapper SSOs, color-coded by taxonomic class based on $g-r$ color only.

0.2 (out of 32776 asteroids with three colors). Their pseudoreflectrance spectra in Fig. 11 (computed using the solar colors of Holmberg et al. 2006, adapted to SkyMapper) agree well with the template spectra of taxonomic classes (DeMeo et al. 2009).

We illustrate the potential of this taxonomic classification in Fig. 12. The photometric classes show clear concentrations in the orbital parameters for different asteroid families. For example, asteroids of the Vesta family (light green points) are concentrated in the inner belt, which is otherwise dominated by asteroids of $S$ complex (red points). $\mathrm{C}$ and $\mathrm{B}$ complexes dominate in the outer belt region, but the Koronis family at 2.9 AU distance shows a clear S-complex taxonomy. It is interesting to note that X-type asteroids (indicated by gray points) are a mixture of $\mathrm{P}, \mathrm{M}$, and E asteroid types in the Tholen classification (Tholen 1989), and are concentrate in the outer belt region associated with the Eos family.

We tested this classification by comparing the SkyMapper classes against previously reported taxonomy from spectroscopy (e.g., Bus \& Binzel 2002; Lazzaro et al. 2004; Binzel et al. 2019; Fornasier et al. 2004, 2014). We found 1,683 SSOs in common and compare their classes in Fig. 13). As expected, rare and peculiar classes such as A, K, L, and Q may be underestimated here and may be misclassified as S. Similarly, there is some confusion between $\mathrm{C} / \mathrm{B}$ and $\mathrm{C} / \mathrm{X}$ classes, which only differ by spectral slope. The values, however, are concentrated on the diagonal of the confusion matrix, showing an overall agreement.

We repeated the same exercise for each SSO with two colors among $(g-r, i-z),(g-i, i-z)$, or $(r-i, i-z)$, accounting for 39063,33299 , and 35498 SSOs, respectively. We present the statistic for all these taxonomic classes in Table 5.

\subsection{Single-color-based taxonomy}

A large number of 117356 SSOs in SkyMapper have only one color: $g-r$. A detailed classification cannot be achieved, but this color can still be used to split asteroids into mafic-silicate-rich and opaque-rich objects. Similarly to the work by (Erasmus et al. 2020), we thus classified the asteroids into S-like and C-like objects (hereafter labeled $S_{1}$ and $C_{1}$ ). The $g-r$ colors of asteroids

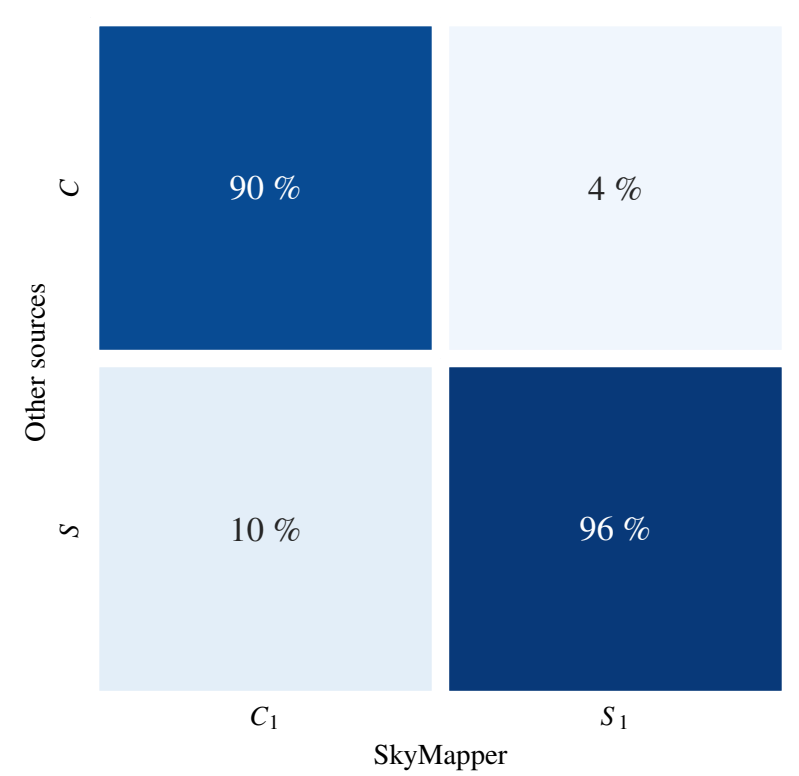

Fig. 16. Confusion matrix of $C$ and $S$ taxonomy complexes of 1129 joint asteroids with taxonomy known from spectra and SkyMapper taxonomy based on $g-r$ colors only.

clearly present a bimodal distribution that is well represented by two normal distributions (Fig. 14) corresponding to the $C_{1}$ (bluer) and $S_{1}$ (redder) classes.

From these two normal distributions, we assigned a probability of belonging to each complex to each asteroid. We then assigned to each object its most probable complex. In this way, we classified 71822 asteroids as a $S_{1}$ type and 40590 as $C_{1}$ type, with the exception of the 4944 asteroids whose probability did not differ by more than $10 \%$. The distribution of $S_{1}$ and $C_{1}$ asteroids also reproduces the known distribution of compositions in the asteroid belt (Fig. 15).

It is interesting to note that even this simplistic taxonomy criterion provides a very good agreement with spectral observations (Fig. 16). 

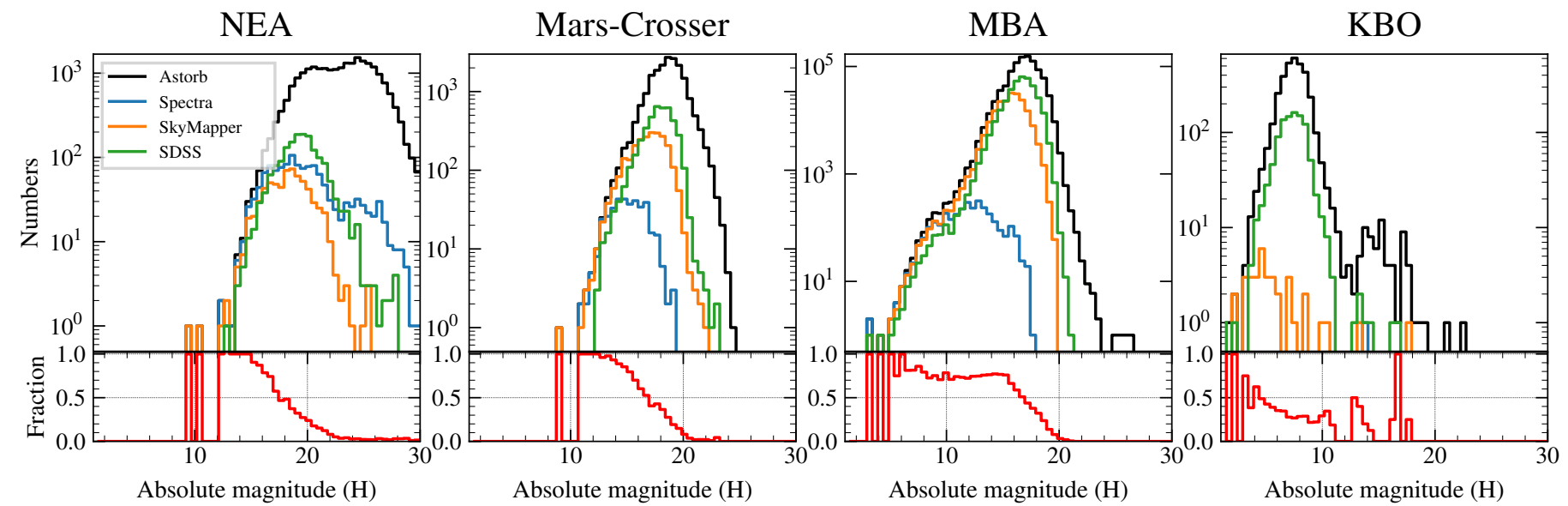

Fig. 17. Comparison of the number of SSOs with either spectra, SDSS colors, or SkyMapper colors, compared with the total number of SSO (taken from astorb) for each of the NEA, Mars-crossers, MBA, and KBO dynamical populations.

\section{Future work}

The present catalog represents the first release of SSOs observed by the SkyMapper Southern Survey, based on the data from its third data release (DR3). These data extend the multifilter photometry provided by the SDSS, which completed its imaging survey in 2009 (although it is still used by the community, see, e.g., Sergeyev \& Carry 2021; Beck \& Poch 2021). SkyMapper is still operating and we plan to release observations of SSOs in upcoming data releases. SkyMapper near-simultaneous acquisition of different filters provides instantaneous determination of colors, which can be used by the community even with the start of operations of the upcoming Legacy Survey of Space and Time (LSST) by the Vera C. Rubin observatory, which will require years to build the phase functions needed to determine SSO colors (Jones et al. 2009; Mahlke et al. 2021).

SkyMapper multifilter observations are rather unique as of today. The observatories that submitted most data to the Minor Planet Center so far this year (2021) are the Catalina Sky Survey, ATLAS (Asteroid Terrestrial-impact Last Alert System), and Pan-STARRS. While Catalina observed the most, it only has a wide optical filter. ATLAS uses two filters and its observing cadence allows for phase functions to be constructed (Mahlke et al. 2021), and a single color can be used for rough taxonomic classification (see Sect. 7.3 and Erasmus et al. 2020). Pan-STARRS has lately contributed to most NEA discoveries, but has relied on $g, r, i$, and a wide $w$ filter, precluding spectral classification as the $z$ filter probes the $1 \mu \mathrm{m}$ band (the most characteristic spectral feature in all major taxonomies since Chapman et al. (1975).

Finally, considering the limiting magnitude of SkyMapper, it is unlikely that a large number of unknown SSOs may be discovered in its images. We plan to proceed with the extraction of known SSOs only in future releases.

\section{Conclusion}

We extracted known SSOs from SkyMapper Southern Survey DR3 images. We applied a suite of filters to minimize contamination. We release a catalog of 880528 individual observations, consisting of 205515 unique known SSOs. The catalog contains the SkyMapper identification, astrometry, photometry, SSO identification, geometry of observation, and taxonomy. Its content is fully described in Appendix A.

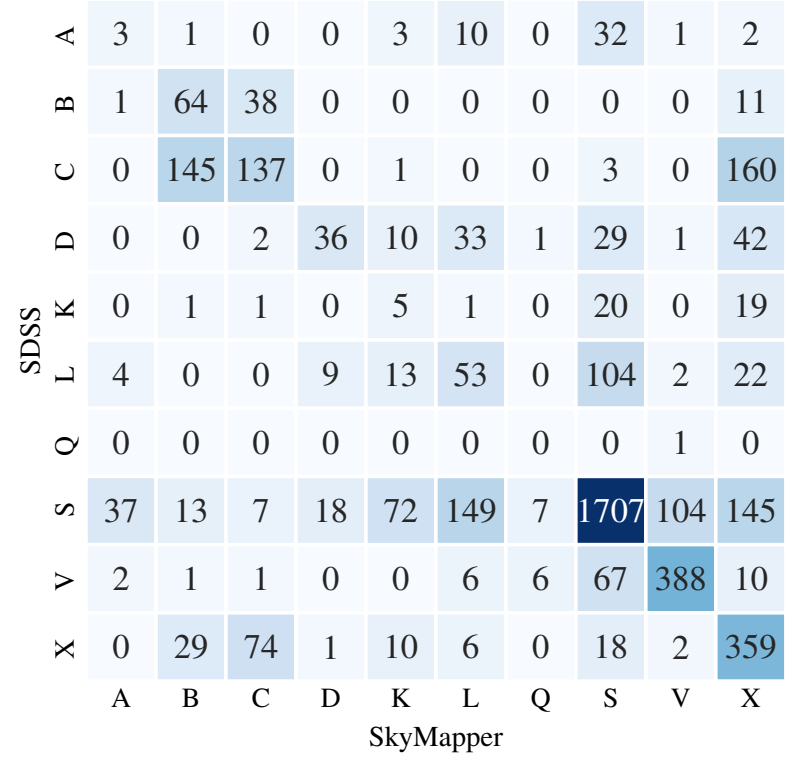

Fig. 18. Confusion matrix of 6965 asteroid taxonomy from the SDSS and SkyMapper with a probability accuracy of more than 0.2

The catalog completeness is estimated to be about $97 \%$ down to $V=18 \mathrm{mag}$, and the purity is estimated to be almost $100 \%$ down to $V=20 \mathrm{mag}$. The present catalog contains photometry of 669 near-Earth asteroids, 2487 Mars-crossers, 196269 MBAs, and 1928 Jupiter trojans.

The SDSS observed 100005 asteroids previously (Sergeyev \& Carry 2021). Combined, the SDSS and SkyMapper data sets account for almost $80 \%$ of all MBAs with an absolute magnitude $(H)$ between 10 and 16, each data set contributing equally. As most of the brighter asteroids $(H<10)$ have spectra, the present SkyMapper release represents a major step toward a completeness of the taxonomic classification of asteroids in the main belt (Fig. 17). The situation is somewhat similar for NEAs and Mars-crossers, with a completeness above $80 \%$ of these two populations down to an absolute magnitude of 16 , although spectral surveys contributed more (e.g., Perna et al. 2018; Devogèle et al. 2019; Binzel et al. 2019). The distant KBOs are the population with the lowest completeness of color characterization, below $50 \%$ (caveat: we did not attempted to 
compile spectra of KBOs here). As SkyMapper is an ongoing survey, this completeness will increase with future releases.

We compared the taxonomy classification of 6965 asteroids in common with the recent SDSS release (Sergeyev \& Carry 2021) and the present SkyMapper catalog, considering only SSOs with a taxomony based on three colors and a probability above 0.2. The confusion matrix presented in Fig. 18 shows a good agreement for the most common complexes such as $\mathrm{S}$, $\mathrm{C}, \mathrm{B}$, and V. Some confusion is present between less prominent classes, in particular, between K, L, A and S. This highlights the strength and the limitation of taxonomic classification based on broadband colors alone.

Acknowledgements. This research has been conducted within the NEOROCKS project, which has received funding from the European Union's Horizon 2020 research and innovation programme under grant agreement No. 870403. CAO was supported by the Australian Research Council (ARC) through Discovery Project DP190100252. SWC acknowledges support from the National Research Foundation of Korea (NRF) grant, No.2020R1A2C3011091, funded by the Korea government (MSIT). We thank J. Berthier, F. Spoto, M. Mahlke for discussions related to the present article. The national facility capability for SkyMapper has been funded through ARC LIEF grant LE130100104 from the Australian Research Council, awarded to the University of Sydney, the Australian Nationa University, Swinburne University of Technology, the University of Queensland the University of Western Australia, the University of Melbourne, Curtin University of Technology, Monash University and the Australian Astronomical Observatory. SkyMapper is owned and operated by The Australian National University's Research School of Astronomy and Astrophysics. The survey data were processed and provided by the SkyMapper Team at ANU. The SkyMapper node of the All-Sky Virtual Observatory (ASVO) is hosted at the Nationa Computational Infrastructure (NCI). Development and support of the SkyMapper node of the ASVO has been funded in part by Astronomy Australia Limited (AAL) and the Australian Government through the Commonwealth's Education Investment Fund (EIF) and National Collaborative Research Infrastructure Strategy (NCRIS), particularly the National eResearch Collaboration Tools and Resources (NeCTAR) and the Australian National Data Service Projects (ANDS). The Pan-STARRS1 Surveys (PS1) and the PS1 public science archive have been made possible through contributions by the Institute for Astronomy, the University of Hawaii, the Pan-STARRS Project Office, the Max-Planck Society and its participating institutes, the Max Planck Institute for Astronomy, Heidelberg and the Max Planck Institute for Extraterrestrial Physics, Garching, The Johns Hopkins University, Durham University, the University of Edinburgh, the Queen's University Belfast, the Harvard-Smithsonian Center for Astrophysics, the Las Cumbres Observatory Global Telescope Network Incorporated, the National Central University of Taiwan, the Space Telescope Science Institute, the National Aeronautics and Space Administration under Grant No. NNX08AR22G issued through the Planetary Science Division of the NASA Science Mission Directorate, the National Science Foundation Grant No. AST-1238877, the University of Maryland, Eotvos Lorand University (ELTE), the Los Alamos National Laboratory, and the Gordon and Betty Moore Foundation. This research made use of the cross-match service provided by CDS, Strasbourg (Pineau et al. 2017), the IMCCE's SkyBoTand Skybot3D VO tools (Berthier et al. 2006, 2016), the JPL Horizons system (Giorgini et al. 1996), the SVO Filter Profile Service (http://svo2.cab.inta-csic.es/theory/fps/) supported from the Spanish MINECO through grant AYA2017-84089 (Rodrigo et al. 2012), and TOPCAT/STILTS (Taylor 2005). Thanks to the developers.

\section{References}

Beck, P., \& Poch, O. 2021, Icarus, 365
Berthier, J., Carry, B., Vachier, F., Eggl, S., \& Santerne, A. 2016, MNRAS, 458, 3394

Berthier, J., Vachier, F., Thuillot, W., et al. 2006, Astronomical Data Analysis Software and Systems XV, C. Gabriel, C. Arviset, D. Ponz, \& S. Enrique ASP Conf. Ser., 458, 3394

Binzel, R. P., DeMeo, F. E., Turtelboom, E. V., et al. 2019, Icarus, 324, 41

Brown, A. G. A., Vallenari, A., Prusti, T., et al. 2018, A\&A, 616, A1

Bus, S. J., \& Binzel, R. P. 2002, Icarus, 158, 146

Carry, B. 2018, A\&A, 609, A113

Carvano, J. M., Hasselmann, P. H., Lazzaro, D., \& Mothé-Diniz, T. 2010, A\&A, 510, A43

Casagrande, L., Wolf, C., Mackey, A. D., et al. 2019, MNRAS, 482, 2770

Chambers, K.C., Magnier, E.A., Metcalfe, N., et al. 2016, ArXiv e-prints [arXiv:1612.05560]

Chapman, C. R., Morrison, D., \& Zellner, B. H. 1975, Icarus, 25, 104

Clement, M. S., Morbidelli, A., Raymond, S. N., \& Kaib, N. A. 2020, MNRAS, 492, L56

DeMeo, F. E., \& Carry, B. 2013, Icarus, 226, 723

DeMeo, F., \& Carry, B. 2014, Nature, 505, 629

DeMeo, F., Binzel, R. P., Slivan, S. M., \& Bus, S. J. 2009, Icarus, 202, 160

DeMeo, F., Binzel, R. P., Carry, B., Polishook, D., \& Moskovitz, N. A. 2014, Icarus, 229, 392

DeMeo, F. E., Polishook, D., Carry, B., et al. 2019, Icarus, 322, 13

Devogèle, M., Moskovitz, N., Thirouin, A., et al. 2019, AJ, 158, 196

Erasmus, N., Navarro-Meza, S., McNeill, A., et al. 2020, ApJS, 247, 13

Fornasier, S., Dotto, E., Marzari, F., et al. 2004, Icarus, 172, 221

Fornasier, S., Lantz, C., Barucci, M. A., \& Lazzarin, M. 2014, Icarus, 233, 163

Giorgini, J. D., Yeomans, D. K., Chamberlin, A. B., et al. 1996, AAS/Div. Planet. Sci. Meeting Abstracts, 28, 25.04

Graves, K., Minton, D., Hirabayashi, M., DeMeo, F., \& Carry, B. 2018, Icarus, 304,162

Holmberg, J., Flynn, C., \& Portinari, L. 2006, MNRAS, 367, 449

Huang, Y., Chen, B. Q., Yuan, H. B., et al. 2019, ApJS, 243, 7

Ivezić, Ž., Tabachnik, S., Rafikov, R., et al. 2001, AJ, 122, 2749

Jenniskens, P., Gabadirwe, M., Yin, Q.-Z., et al. 2021, Meteorit. Planet. Sci., 56, 844

Jones, R. L., Chesley, S. R., Connolly, A. J., et al. 2009, Earth Moon Planets, 105,101

Lazzaro, D., Angeli, C. A., Carvano, J. M., et al. 2004, Icarus, 172, 179

Levison, H. F., Bottke, W. F., Gounelle, M., et al. 2009, Nature, 460, 364

Mahlke, M., Carry, B., \& Denneau, L. 2021, Icarus, 354

Morbidelli, A., Walsh, K. J., O’Brien, D. P., Minton, D. A., \& Bottke, W. F. 2015,

The Dynamical Evolution of the Asteroid Belt, 493

Nesvorný, D., Jedicke, R., Whiteley, R. J., \& Ivezić, Ž. 2005, Icarus, 173, 132

Oke, J. B., \& Gunn, J. E. 1983, ApJ, 266, 713

Onken, C. A., Wolf, C., Bessell, M. S., et al. 2019, PASA, 36

Parker, A., Ivezić, Ž., Jurić, M., et al. 2008, Icarus, 198, 138

Perna, D., Barucci, M. A., Fulchignoni, M., et al. 2018, Planet Space Sci., 157, 82

Pickles, A. J. 1998, PASP, 110, 863

Pineau, F. X., Derriere, S., Motch, C., et al. 2017, A\&A, 597, A89

Popescu, M., Licandro, J., Morate, D., et al. 2016, A\&A, 591, A115

Rodrigo, C., Solano, E., Bayo, A., \& Rodrigo, C. 2012, SVO Filter Profile Service Version 1.0, Tech. rep.

Sergeyev, A. V., \& Carry, B. 2021, A\&A, 652, A59

Siegel, A. F. 1982, Biometrika, 69, 242

Szabó, G. M., Ivezić, Ž., Jurić, M., Lupton, R., \& Kiss, L. L. 2004, MNRAS, 348,987

Taylor, M. B. 2005, Astronomical Data Analysis Software and Systems XIV, P. Shopbell, M. Britton, \& R. Ebert, ASP Conf. Ser., 347, 29

Tholen, D. J. 1989, Asteroids II, R. P. Binzel, T. Gehrels, \& M. S. Matthews, 1139

Wolf, C., Onken, C. A., Luvaul, L. C., et al. 2018, PASA, 35, 10 


\section{Appendix A: Description of catalogs}

We describe here the three catalogs of SSOs we release. The detection catalog (Table A.1) contains all the information for each observation. (mid-observing time, coordinates, etc.). The color catalog contains all the measured SSO colors (Table A.2), while the object catalog (Table A.3) contains a single entry per SSOs, with its average colors.

Table A.4 contains the estimated taxonomy and orbital elements of asteroids. The most probable taxonomy depends on the color priority in the follow sequence: $g-r, g-i, i-z$ colors have the first priority, two colors $(g-r, i-z),(g-i, i-z),(r-i, i-z)$ have the priority 2,3 , and 4 , and consequently $g-r$, have the color priority 5 .

Table A.1. Description of the SkyMapper catalog of SSO observations.

\begin{tabular}{|c|c|c|c|}
\hline ID & Name & Unit & Description \\
\hline 1 & source & & Source unique identifier \\
\hline 2 & frame & & Image unique identifier \\
\hline 3 & JD & day & Julian Date of observation \\
\hline 4 & filter & & Filter name $(u, v, g, r, i, z)$ \\
\hline 5 & exptime & S & Exposure time \\
\hline 6 & ra & $\operatorname{deg}$ & J2000 Right Ascension \\
\hline 7 & dec & deg & J2000 Declination \\
\hline 8 & psfMag & mag & PSF magnitude \\
\hline 9 & psfMagErr & mag & PSF magnitude uncertainty \\
\hline 10 & petroMag & mag & Petrosian magnitude \\
\hline 11 & petroMagErr & mag & Petrosian magnitude uncertainty \\
\hline 12 & number & & SSO IAU number \\
\hline 13 & name & & SSO IAU designation \\
\hline 14 & dynclass & & SSO dynamical class \\
\hline 15 & ra_rate & $" / \mathrm{h}$ & RAcos(DEC) rate of motion \\
\hline 16 & dec_rate & $" / \mathrm{h}$ & DEC rate \\
\hline 17 & V & mag & Predicted visual magnitude \\
\hline
\end{tabular}

Table A.2. Description of the color catalog extracted from all SkyMapper SSO observations.

\begin{tabular}{cccc}
\hline \hline ID & Name & Unit & Description \\
\hline 1 & number & & SSO IAU number \\
2 & name & & SSO IAU designation \\
3 & JD & day & Average epoch of observation \\
4 & color & & Name of color (e.g., $g-r)$ \\
5 & dmag & mag & Value of the color \\
6 & edmag & mag & Uncertainty on the color \\
7 & dmjd & day & Delay between filters \\
\hline
\end{tabular}

Table A.3. Description of the catalog of measured weighted mean SSO colors.

\begin{tabular}{lccc}
\hline \hline ID & Name & Unit & Description \\
\hline 1 & number & SSO IAU number \\
2 & name & & SSO IAU designation \\
4 & color & & Name of color (e.g., $g-r)$ \\
5 & wdmag & mag & Weighted value of the color \\
6 & var & mag & Weighted uncertainty of the color measurements \\
7 & n & & Number of color measurements, 0 - computed \\
6 & emag & mag & Mean value of the color uncertainties \\
7 & dmjd & day & Mean time difference between color measurements \\
\hline
\end{tabular}

Table A.4. Description of individual SkyMapper SSO color catalog (measured and computed) and their taxonomy.

\begin{tabular}{|c|c|c|c|}
\hline ID & Name & Unit & Description \\
\hline 1 & number & & SSO IAU number \\
\hline 2 & name & & SSO IAU designation \\
\hline 3 & dynclass & & SSO dynamic class \\
\hline $4-18$ & $\mathrm{~m}\left[{ }^{*}\right.$ color list $]$ & mag & $\begin{array}{l}\text { Set of the color magnitude } \\
\text { values }\end{array}$ \\
\hline $19-33$ & $\mathrm{e}\left[{ }^{*}\right.$ color list $]$ & mag & Set of the color uncertainties \\
\hline $34-48$ & $\mathrm{~d}\left[{ }^{*}\right.$ color list $]$ & day & $\begin{array}{l}\text { sSet of time values between } \\
\text { observations }\end{array}$ \\
\hline $49-59$ & $\begin{array}{l}\mathrm{p}[\mathrm{C}, \mathrm{S}, \mathrm{B}, \mathrm{L} \\
\mathrm{D}, \mathrm{K}, \mathrm{X}, \mathrm{Q} \\
\mathrm{V}, \mathrm{A}, \mathrm{U}]\end{array}$ & & $\begin{array}{l}\text { Probability of the complex } \\
\text { values }\end{array}$ \\
\hline 60 & complex & & Most probably complex \\
\hline 61 & pcomplex & & $\begin{array}{l}\text { Probability value of the com- } \\
\text { plex }\end{array}$ \\
\hline 62 & complex 1 & & $\begin{array}{l}\text { First the most probably com- } \\
\text { plex }\end{array}$ \\
\hline 63 & complex2 & & $\begin{array}{l}\text { Second the most probably } \\
\text { complex }\end{array}$ \\
\hline 64 & pcomplex 1 & & $\begin{array}{l}\text { Probability value of first the } \\
\text { most probably complex }\end{array}$ \\
\hline 65 & pcomplex2 & & $\begin{array}{l}\text { Probability value of second } \\
\text { the most probably complex }\end{array}$ \\
\hline 66 & $\mathrm{nc}$ & & $\begin{array}{l}\text { The taxonomy priority num- } \\
\text { ber based on used colors (see } \\
\text { description in Appendix A) }\end{array}$ \\
\hline & $\pi \quad r$ & & 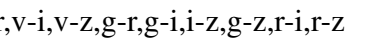 \\
\hline
\end{tabular}




\section{Appendix B: Multi-color taxonomy}

We present here the color distribution, pseudo-reflectance, confusion matrix, orbital distribution, and taxonomy boundaries of asteroids with two colors.
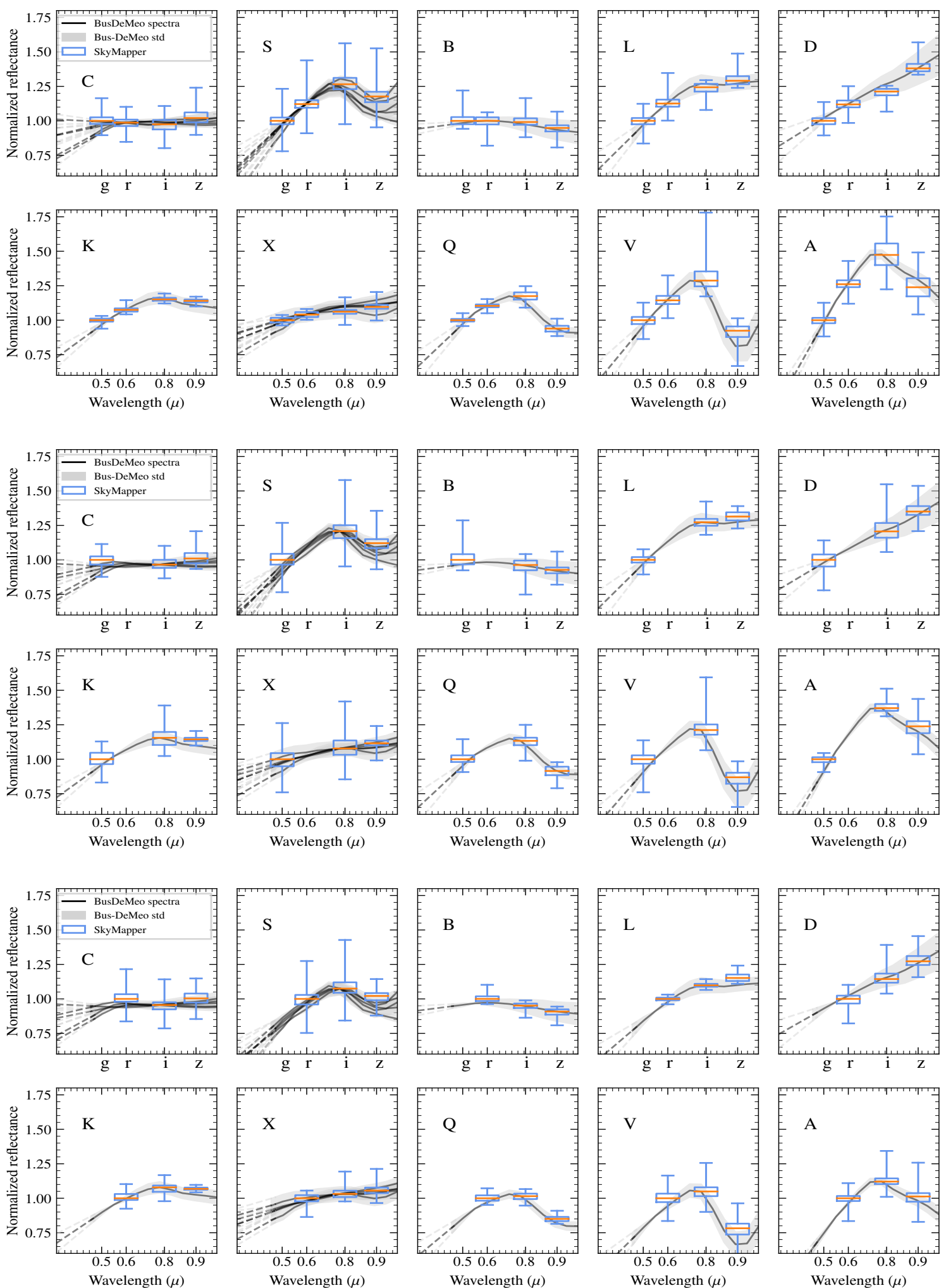

Fig. B.1. Grouped by taxonomic class pseudo-reflectance spectra of asteroids based on two-color taxonomy. Top: $g-r, i-z$ colors, middle: $g-i$, $i-z$ colors, and bottom: $r-i, i-z$ colors. We indicate the average wavelength of each filter in the lower plots. The distribution of values for each band is represented by whiskers (95\% extrema, and the 25, 50, and 75\% quartiles). For each, we also represent the associated template spectra of the Bus-DeMeo taxonomy (DeMeo et al. 2009). 
A. V. Sergeyev et al.: Multifilter photometry of Solar System objects from the SkyMapper Southern Survey
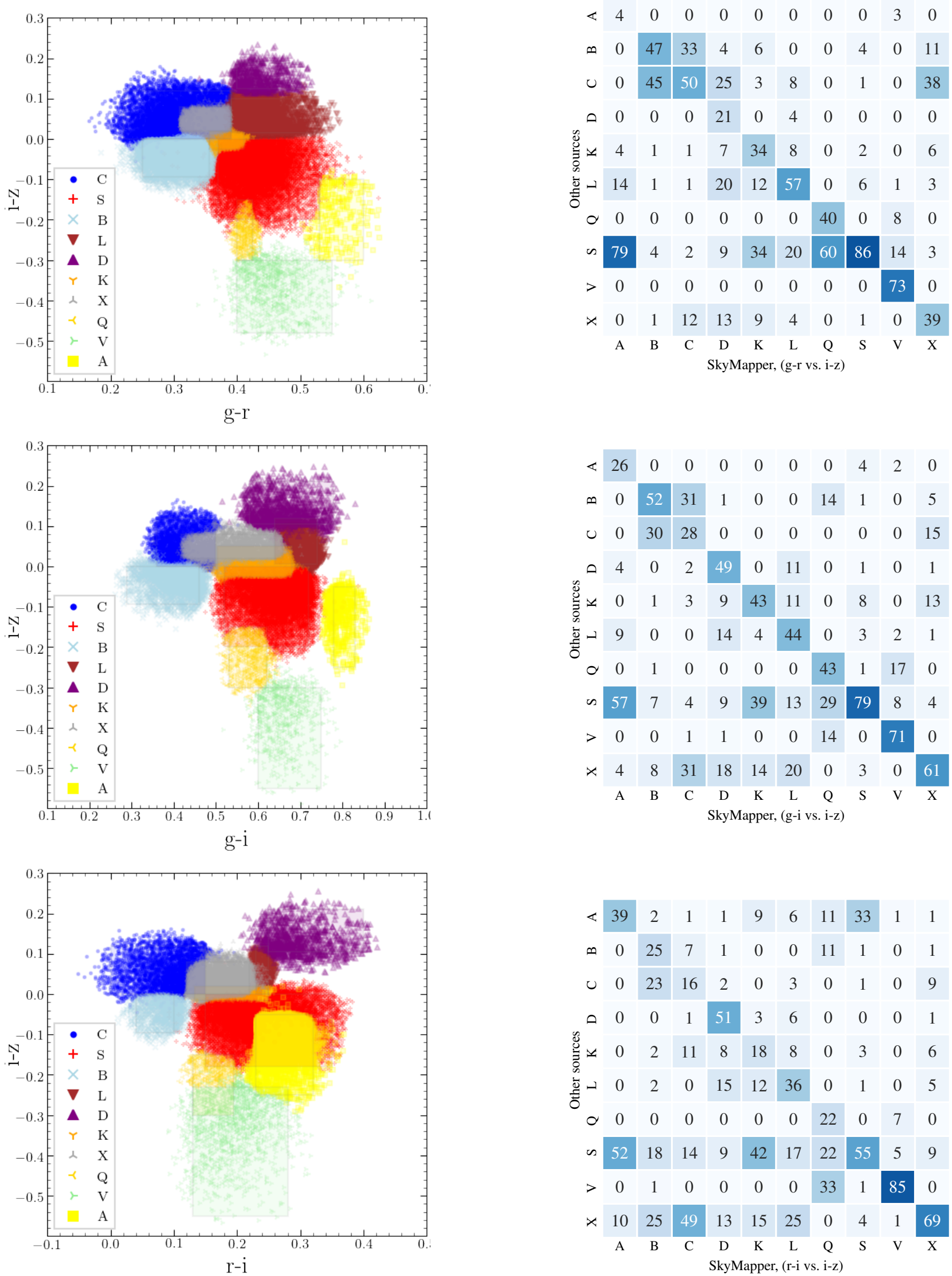

Fig. B.2. Taxonomy of SkyMapper asteroids with a probability value more than 0.2 with two (top: $g-r, i-z$, middle: $g-i, i-z$, and bottom: $r-i, i-z$ ) colors. Boxes show the boundaries of the taxonomic classes. Color points mark an individual asteroid.

Fig. B.3. Confusion matrices of asteroids taxonomy based on (top: $g-r$, $i-z$, middle: $g-i, i-z$, and bottom: $r-i, i-z)$ SkyMapper color opposite taxonomy from published spectral observations. The values are reported in percent. 

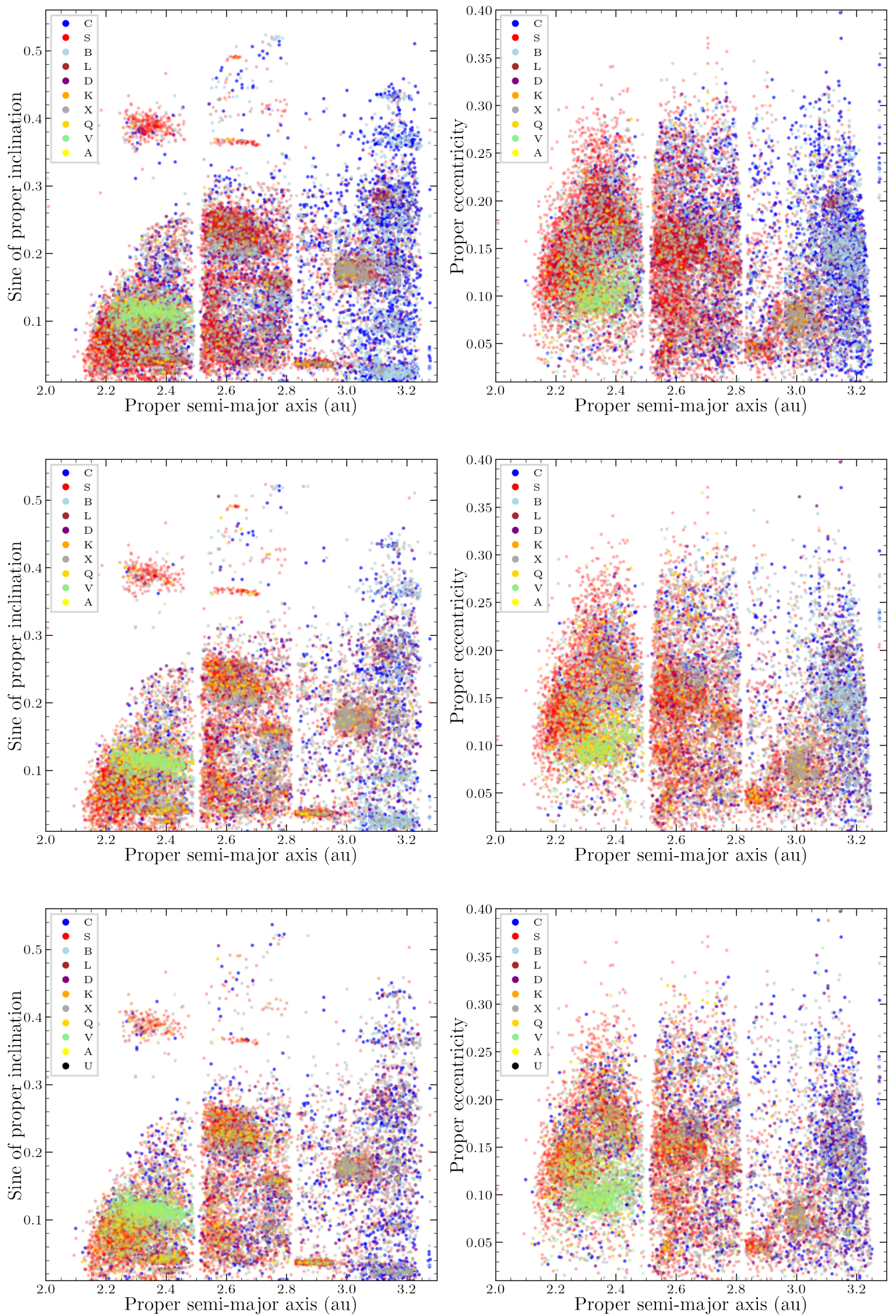

Fig. B.4. Orbital distribution of the SkyMapper SSOs, color-coded by taxonomic class based on their (top: $g-r, i-z$, middle: $g-i, i-z$, and bottom: $r-i, i-z)$ colors. 
A. V. Sergeyev et al.: Multifilter photometry of Solar System objects from the SkyMapper Southern Survey

Table B.1. Boundaries of taxonomy complexes in g-r, g-i, i-z color space.

\begin{tabular}{ccccccc}
\hline \hline complex & $g r_{\min }$ & $g r_{\max }$ & $i z_{\min }$ & $i z_{\max }$ & $g i_{\min }$ & $g i_{\max }$ \\
\hline $\mathrm{C}$ & 0.220 & 0.350 & 0.000 & 0.120 & 0.360 & 0.50 \\
$\mathrm{~S}$ & 0.370 & 0.530 & -0.200 & 0.000 & 0.620 & 0.73 \\
$\mathrm{~B}$ & 0.250 & 0.356 & -0.094 & 0.001 & 0.288 & 0.46 \\
$\mathrm{~L}$ & 0.391 & 0.527 & 0.015 & 0.120 & 0.650 & 0.78 \\
$\mathrm{D}$ & 0.400 & 0.500 & 0.105 & 0.210 & 0.650 & 0.80 \\
$\mathrm{~K}$ & 0.370 & 0.430 & -0.015 & 0.020 & 0.503 & 0.68 \\
$\mathrm{X}$ & 0.280 & 0.400 & 0.000 & 0.080 & 0.400 & 0.62 \\
$\mathrm{Q}$ & 0.400 & 0.425 & -0.300 & -0.150 & 0.530 & 0.62 \\
$\mathrm{~V}$ & 0.350 & 0.600 & -0.550 & -0.230 & 0.520 & 0.75 \\
$\mathrm{~A}$ & 0.459 & 0.595 & -0.250 & -0.050 & 0.780 & 0.83 \\
\hline
\end{tabular}

Table B.2. Boundaries of taxonomy complexes in g-r, i-z color space.

\begin{tabular}{ccccc}
\hline \hline complex & $g r_{\min }$ & $g r_{\max }$ & $i z_{\min }$ & $i z_{\max }$ \\
\hline $\mathrm{C}$ & 0.220 & 0.391 & 0.000 & 0.120 \\
$\mathrm{~S}$ & 0.370 & 0.530 & -0.200 & 0.000 \\
$\mathrm{~B}$ & 0.250 & 0.356 & -0.094 & 0.001 \\
$\mathrm{~L}$ & 0.391 & 0.527 & 0.015 & 0.100 \\
$\mathrm{D}$ & 0.400 & 0.500 & 0.125 & 0.190 \\
$\mathrm{~K}$ & 0.356 & 0.411 & -0.025 & 0.020 \\
$\mathrm{X}$ & 0.309 & 0.391 & 0.020 & 0.080 \\
$\mathrm{Q}$ & 0.400 & 0.425 & -0.300 & -0.150 \\
$\mathrm{~V}$ & 0.400 & 0.550 & -0.480 & -0.300 \\
$\mathrm{~A}$ & 0.530 & 0.600 & -0.300 & -0.100 \\
\hline
\end{tabular}

Table B.3. Boundaries of taxonomy complexes in g-i, i-z color space.

\begin{tabular}{ccccc}
\hline \hline complex & $g i_{\min }$ & $g i_{\max }$ & $i z_{\min }$ & $i z_{\max }$ \\
\hline $\mathrm{C}$ & 0.360 & 0.500 & 0.000 & 0.120 \\
$\mathrm{~S}$ & 0.520 & 0.720 & -0.200 & 0.000 \\
$\mathrm{~B}$ & 0.288 & 0.460 & -0.094 & 0.001 \\
$\mathrm{~L}$ & 0.640 & 0.740 & 0.005 & 0.120 \\
$\mathrm{D}$ & 0.580 & 0.780 & 0.105 & 0.210 \\
$\mathrm{~K}$ & 0.503 & 0.680 & -0.025 & 0.050 \\
$\mathrm{X}$ & 0.417 & 0.658 & 0.020 & 0.080 \\
$\mathrm{Q}$ & 0.530 & 0.620 & -0.300 & -0.150 \\
$\mathrm{~V}$ & 0.600 & 0.750 & -0.550 & -0.300 \\
$\mathrm{~A}$ & 0.780 & 0.830 & -0.250 & -0.050 \\
\hline
\end{tabular}

Table B.4. Boundaries of taxonomy complexes in $\mathrm{r}-\mathrm{i}$, i-z color space.

\begin{tabular}{ccccc}
\hline \hline complex & $r i_{\min }$ & $r i_{\max }$ & $i z_{\min }$ & $i z_{\max }$ \\
\hline $\mathrm{C}$ & 0.000 & 0.150 & 0.000 & 0.120 \\
$\mathrm{~S}$ & 0.150 & 0.350 & -0.180 & 0.000 \\
$\mathrm{~B}$ & 0.040 & 0.100 & -0.095 & 0.001 \\
$\mathrm{~L}$ & 0.230 & 0.250 & 0.000 & 0.120 \\
$\mathrm{D}$ & 0.250 & 0.400 & 0.105 & 0.210 \\
$\mathrm{~K}$ & 0.133 & 0.250 & -0.015 & 0.020 \\
$\mathrm{X}$ & 0.120 & 0.220 & 0.000 & 0.080 \\
$\mathrm{Q}$ & 0.130 & 0.195 & -0.300 & -0.150 \\
$\mathrm{~V}$ & 0.130 & 0.280 & -0.550 & -0.230 \\
$\mathrm{~A}$ & 0.230 & 0.320 & -0.250 & -0.050 \\
\hline
\end{tabular}

